\section{LAS AULAS ESCOLARES COMO ESCENARIO DE FORMACIÓN EN CIUDADANÍA ${ }^{12}$}

\section{EDUCATION FOR THE CITIZENSHIP FROM THE SCHOOL CLASSROOMS.}

\section{José Rubén Castillo García ${ }^{3}$}

Palabras Clave: El mundo escolar, la ciudadanía, retos del mundo escolar, la educación para la ciudadanía, la ciudadanía en los jóvenes estudiantes.

Key words: The school world, the citizenship, the challenges of the school world, the education for the citizenship, the citizenship in the young students

\section{ABSTRACT}

This document raises from a preamble and two topics that develop the body of the contents:

1. Challenges of culture, and 2 challenges of the school world in terms of citizenship.

The preamble locates the social daily practices of the young students in the educational environment which derives in the need to look at the different rhythms that experience the school institutions from social, cultural, economic and political dynamics.

Also the changes that affect the processes of construction of the subjectivity of the students are considered specifically in the environments of the culture and of the configuration of the citizenship
1 La base de esta ponencia es un capítulo de la investigación denominada "Configuración de ciudadanías juveniles en la vida cotidiana de estudiantes universitarios de Manizales", presentada por el autor para optar al título de Doctor en Ciencias Sociales, niñez y juventud. Universidad de Manizales - CINDE, 2006.

Este texto ha sido construido a partir de la ponencia aceptada por el Comité Científico del Primer PreCongreso Mundial de los Derechos de la Niñez y Adolescencia, y presentada en el evento celebrado durante los días 19,20 y 21 de septiembre de 2007 en la Universidad de Puerto Rico en Arecibo, con el nombre de "Educación para la ciudadanía desde las aulas escolares" $\mathrm{Y}$ aceptada en el III Congreso Mundial sobre derechos de la niñez y adolescencia en Barcelona a celebrarse los días 14 a 19 de noviembre de 2007

2 La vinculación del autor del texto al programa doctoral en Ciencias sociales, niñez y juventud, y la consiguiente aceptación de la propuesta de investigación que serviría de tesis, fue aprobada por el Comité de Rectoría de la Universidad Autónoma de Manizales mediante comunicación del 28 de julio de 2000. La investigación se desarrolló entre agosto de 2000 y noviembre de 2006.

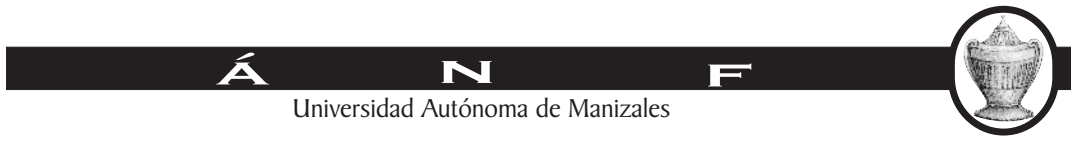


The challenges that come from the culture, take us to the stage of the socialization and from there to the school institution seen as the space where the dialogues, exchanges, confrontations are carried out and specially the production of subjectivities.

Then, it invites us to look at the school world in terms of the citizenship and proposes to us several aspects from which it will look at the role of the educational institutions in this field: the historical condition, as a form to exercise the social issue, the social thing and the cultural thing, the rules, the rights and duties, the possibilities and opportunities, and the challenges towards future the school world must face to contribute in the configuration of the citizens.

We think that in this way, we take part of the reflection about the new environments in which citizenship takes place and is performed, in this case, from the educational view and facing it as a matter of human development in the liberating perspective of the human kind.

\section{RESUMEN}

Este documento plantea tres tópicos, un preámbulo y dos desafíos, que buscan ubicar el desarrollo de los contenidos en el marco de la cultura, $y$ del mundo escolar en términos de la ciudadanía.

El preámbulo, contextúa a los jóvenes estudiantes en el ámbito de sus prácticas sociales cotidianas, e invita a pensar en los diferentes ritmos que se vivencian en las instituciones escolares y en la necesidad de que se adecúen a las demandas de la realidad para que puedan contribuir adecuadamente a la configuración de las subjetividades de los estudiantes, específicamente en los ámbitos de la formación en la ciudadanía.

Los desafíos que emanan de la cultura, nos llevan a ver la institución escolar como un escenario de socialización, desde el cual se efectúan los diálogos, intercambios, negociaciones, confrontaciones y ante todo de la producción de subjetividades.

Luego, el texto nos invita a mirar el mundo escolar en términos de formación para la ciudadanía y nos propone varios aspectos desde los cuales se debe mirar el papel de las instituciones educativas en este
3 Docente investigador de la Universidad Autónoma de Manizales y Docente del Centro Auxiliar de Servicios Docentes -CASD- de Manizales, Trabajador Social, Magíster en Desarrollo Educativo y Social y Doctor en ciencias sociales, niñez y juventud, miembro del grupo de investigación en Ética y Política y coordinador de la línea de investigación en Competencias Ciudadanas de la Universidad Autónoma de Manizales. Dirección e l e c t r ó n i c a : jorca@autonoma.edu.co y jorca53@gmail.com

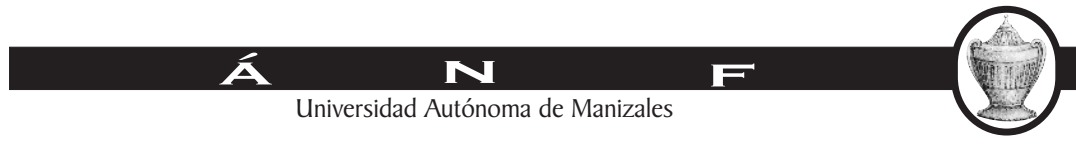


campo: la condición histórica, como forma de ejercer la socialidad, lo social y lo cultural, la normatividad, los derechos y deberes, las posibilidades y oportunidades, y los retos que hacia futuro deben atender estas instituciones para aportar a la configuración de los ciudadanos.

Consideramos que de esta manera participamos de la reflexión acerca de los nuevos ambientes en los cuales se produce y se ejerce la ciudadanía, en este caso, desde la educación y orientándola como un asunto de desarrollo humano en la perspectiva liberadora de los seres humanos.

\section{PREÁMBULO}

Para Rossana Reguillo (2000: c.1), los jóvenes estudiantes son los denominados "incorporados", ésto porque muchas de sus prácticas cotidianas se escenifican en las instituciones escolares. En mi caso, centro la mirada en los "incorporados" que actúan cotidianamente en el ámbito educativo, y pienso, que ellos tienen especificidades en sus formas de ser, pensar, sentir y actuar.

Me preocupa que las instituciones educativas, poco tienen en cuenta los cambios y dinámicas que se presentan en las sociedades, cambios que ocurren a una velocidad insospechada, mientras la realidad se transforma con gran rapidez. La institución escolar avanza a un ritmo lento, y por ende, se ha visto incapaz de adecuar sus contenidos y procedimientos a las demandas de dicha realidad. Esta diferencia de ritmos de transformación produce descontextualización en las instituciones educativas con respecto de lo social, cultural, económico y político.

En el presente texto nos respaldamos, entre otros, en los siguientes supuestos:

Hay divorcio en el tipo de lenguajes que se utilizan en los procesos de enseñanza, dado que los jóvenes estudiantes privilegian la oralidad y menos la escritura, mientras que los docentes dejan de lado las sensibilidades audiovisuales o las utilizan sólo como recursos que apoyan las prácticas pedagógicas tradicionales. 
La escuela, se ha orientado, entre muchas y diversas funciones, a la socialización de los niños y los jóvenes. Desde su aparición asume un papel clave en la formación de sujetos sociales, por tanto, su función no se restringe a la transmisión de los saberes acumulados. Esos saberes se utilizan bien sea para reproducir la fuerza de trabajo, en cuyo caso formaría sólo profesionales, o para formar ciudadanos en general., es decir, forma personas para que se desenvuelvan en los diferentes ámbitos sociales.

La escuela ha contribuido a la emergencia de la juventud como paradigma cultural, puesto que en ella se dan cita grupos de jóvenes que quieren lograr diversas cosas: aprender a trabajar como lo hacen sus padres, crear, divertirse, relacionarse y convivir con otras personas, ubicarse de manera propia en el planeta, contar sus historias, reír y llorar a su manera. Así, ésta institución ejerce como un escenario donde hacen presencia los significados y sentidos de vida.

Los contenidos, vivencias y experiencias que se construyen en la escuela dependen en gran medida de las nuevas y diversas circunstancias socio-históricas: la globalización, la mundialización de la cultura, la dinámica en los procesos de comunicación, la modificación de los paradigmas sociales, entre otras. De ello derivan las diversas prácticas que se dan en ella como institución.

El contexto social, ejerce como circunstancia exógena a la escuela y evidencia las modificaciones que se perciben en las estructuras políticas, económicas, sociales, y culturales. Éstas inciden en la generación de significados, en los intercambios de sentidos y en la difusión de los mismos, además afectan notablemente los procesos de construcción de las subjetividades de los sujetos que viven los mundos escolares.

El contexto institucional, asume como circunstancia endógena y se manifiesta en asuntos relacionados con los objetivos, finalidades, procedimientos, medios e intencionalidades que se utilizan en la escuela para que ésta pueda cumplir con el compromiso de formación de los sujetos sociales que puedan responder a las demandas de las sociedades contemporáneas. Al respecto, se evidencian serias preocupaciones relacionadas con los resultados que trae la masificación 
de los estudiantes, puesto que la cantidad puede ir en detrimento de la calidad, y las relaciones de poder entre los diversos sectores de la comunidad educativa.

Es de tener en cuenta que hoy en día, (Tenti Fanfanil, citando a Tudesco, 2000) los procesos de formación escolar no llevan al ascenso social de sus egresados, dado que una vez culminados y certificados sus estudios, no tienen la garantía de vinculación laboral. La educación en la actualidad no es selectiva puesto que no da cuenta de la cualificación de los sujetos, ni establece parámetros para definir a los más capaces. Además, la retención escolar es más un asunto de prevención social que de garantizar los derechos de los ciudadanos.

Lo anterior, permite evidenciar algunos desafíos que tienen que ver con dos esferas de la realidad en que se desenvuelven los jóvenes: en primer lugar, la cultura asumida como el marco donde se dan las formas de percibir y de interpretar los mundos en que viven como estudiantes, y en segundo lugar, la ciudadanía, pensada como la configuración social de los sujetos, en razón de las relaciones de convivencia que resultan de las prácticas sociales que devienen de la vida escolar. A ellos vamos a referirnos en adelante.

\section{Desafíos que se generan en las aulas escolares en el campo de la cultura}

Los sujetos humanos somos resultado de los procesos sociales que nos han permitido construir las vidas particulares. A través de la socialización adquirimos los modelos de interpretación de la realidad y a partir de éstos hemos elaborado nuestras propias formas de ser. Esto indica que los jóvenes estudiantes llevan a las aulas, como parte de su bagaje, las expresiones de sus modos de ver el mundo, de vivenciarlo, de interpretarlo y de significarlo, dado que son portadores de conocimientos, valores y actitudes que los constituyen como seres culturales.

En muchos casos, existe un abismo entre las vivencias que se proponen desde las instituciones escolares y la vida cotidiana de los jóvenes, ambas instancias transitan por caminos alejados. Los ideales institucionales de la escuela poco se relacionan con los sentidos de vida de los estudiantes.

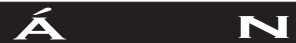

Universidad Autónoma de Manizales 
Se da como un hecho que la vida cotidiana escolar es un escenario en el cual hacen presencia los diferentes sentidos de vida de los sujetos que hacen parte de la comunidad educativa, tanto de los estudiantes como de los maestros y de las familias. Al respecto Sanín (1998: 7) considera que la institución escolar cumple una función importante en los procesos de intercambio de experiencias entre los diversos actores que participan de ella. De esta manera, al menos en su proyecto, los mundos de la escolaridad se convierten en escenarios donde florecen las diferencias, los intercambios, los diálogos, las contradicciones, los conflictos y donde se negocian los diferentes sentidos de vida de distintas maneras:

... la escuela debe entenderse como un lugar donde se estimule el diálogo intergeneracional, que ofrezca tiempos para que se produzcan aprendizajes para la vida en sociedad, un espacio de producción y socialización de la cultura y por ende, y a pesar de todas las transformaciones que han ocurrido en los escenarios de socialización, un lugar y un tiempo privilegiados donde viven y se aprenden las normas y valores que hacen posible la vida en sociedad.

En este mismo sentido, Rossana Reguillo (2000: 20, citando a Mead, 1969) señala que las relaciones intergeneracionales contribuyen a configurar las culturas propias de los mundos juveniles. Según ella, la reproducción sociocultural expresa tres tipos de culturas: la posfigurativa, la cofigurativa y la prefigurativa. La primera se ancla en el pasado, en la tradición y se refiere a las formas en que los niños aprenden de sus mayores; la segunda, pensada como cultura de la modernidad, considera que los niños y los adultos aprenden de sus pares; y en la última, los adultos quiénes aprenden de los niños.

A partir de sus vivencias, experiencias y sentidos de vida, los jóvenes se ponen en contacto con los demás, se organizan y negocian sus sueños e ilusiones. $Y$ el escenario educativo es uno de los puntos de encuentro donde forjan los mundos que consideran posibles. Esos encuentros son mediados por los procesos de comunicación que se establecen entre los diferentes actores que participan en las prácticas educativas. Esas interacciones se dan a partir de los mundos de significado que poseen, de tal manera que lo que realmente se produce son intercambios y negociaciones entre subjetividades.

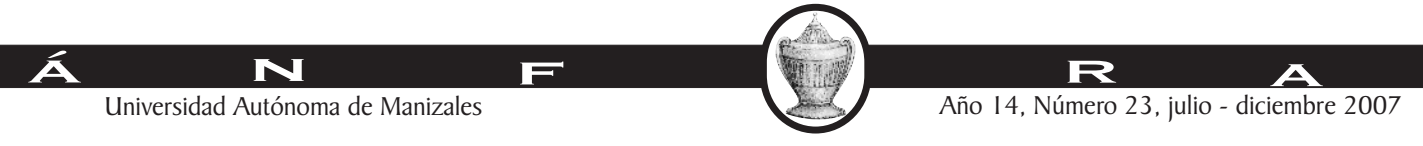


Los procesos comunicativos originados en las prácticas educativas propician la inserción de los jóvenes en la vida pública, en la medida en que tienen la posibilidad de reconocer a los demás y hacerse reconocer por ellos, de acceder a los contextos globales, de identificar otras formas de expresarse ante el mundo, lo cual les permite acceder a diferentes modelos de interpretación de la realidad como resultado del hecho de compartir la vida junto a otros.

De lo dicho, se puede colegir que las instituciones escolares ofrecen importantes posibilidades para que los diferentes actores que participan de la vida académica se configuren como ciudadanos, dado que las prácticas educativas se anclan en los procesos de comunicación, las relaciones sociales, la convivencia y otras consideraciones que llevan a los sujetos a integrarse a la vida de los colectivos.

Veamos lo que ocurre en términos de las prácticas sociales ciudadanas.

\section{Desafíos del mundo escolar en términos de la ciudadanía ${ }^{4}$}

Recordemos que la ciudadanía se refiere a las formas de vinculación, asimilación y de inmersión de los sujetos a las sociedades. La calidad de ciudadanos se deriva de los procesos sociales, y dicha calidad la alcanzan personas que actúan en relación con los otros en el marco de sociedades establecidas y formalizadas. Cuando estas organizaciones sociales los reconocen como ciudadanos, les permiten ciertas prebendas y prerrogativas.

La ciudadanía está referida a las posibilidades que tienen los sujetos para incidir en el funcionamiento de las sociedades. Ha adquirido diversas connotaciones a lo largo de la historia, pero en el fondo se refiere a la vinculación de los ciudadanos a los colectivos, al reconocimiento de sus derechos y a las posibilidades de participación en la toma de decisiones de la comunidad en la cual ejercen dicha calidad. Ésta calidad de los ciudadanos cobra sentido en referencia a varias condiciones, veamos algunas de ellas:

\subsection{La condición histórica de la ciudadanía}

Se da como un hecho que en la actualidad existen nuevas formas de producir y de transmitir los saberes, nuevos lenguajes y modelos de
4.Cfr. CASTILLO, J. R. (2003), p. 115 a 143
A N

Universidad Autónoma de Manizales
$F$

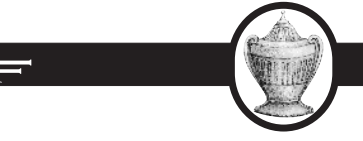


comunicación que a su vez tienen presencia en la escuela. Gimeno Sacristán (2001, p. 158) señala que:

Los nuevos retos que emergen de la globalización exigen nuevos referentes de pensamiento que permitan interpretar las relaciones sociales y considerar la existencia de nuevas perspectivas de generación de cultura. Estos exigen que la ciudadanía se reconstruya a partir de los marcos políticos, económicos y culturales del mundo globalizado. De esta manera, las posibilidades, oportunidades y limitaciones que se originan en el acceso a la Internet, a la educación a distancia y a otras tecnologías virtuales, condicionan notablemente la calidad de los ciudadanos, entre ellos los estudiantes.

Al respecto, valga inferir que las condiciones histórico sociales y las expresiones culturales propias de cada sociedad llevan a que la vida académica sea un espacio donde se manifiestan las nuevas demandas de las sociedades actuales, que implican la necesidad de pensar que hay otras expresiones de ciudadanía, diferentes a las que hasta este momento se conocen. Apoyados en Durston J. (p. 3), podemos afirmar que la aparición y consolidación de nuevas formas de producción económica basadas en la información y el conocimiento, regidas más por el mercado que por el Estado, exige nuevas definiciones del contenido del concepto de ciudadanía, y de las nuevas formas institucionales de relacionarse unas personas con otras y con los colectivos.

\subsection{La ciudadanía como una forma de ejercer la socialidad}

En el escenario escolar, manifestamos diferentes maneras de vincularnos a la dinámica de la vida, de hacer uso de nuestras capacidades, habilidades y destrezas para comunicarnos y relacionarnos con los demás, esto es lo que denominamos socialidad, es decir, la potencialidad de llegar a ser miembros de la sociedad.

En la escuela se construyen discursos que permiten comprender las condiciones de existencia de los estudiantes y sus perspectivas de participar en los desarrollos de los asuntos colectivos. Allí se accede a la tradición ciudadana y a la vivencia de prácticas sociales que pueden o no ser democráticas. En los procesos de vinculación de los individuos a la

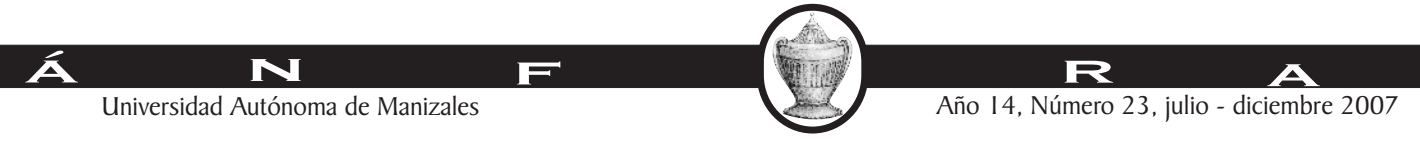


sociedad se identifican espacios y mecanismos de participación y los instrumentos que pueden ser utilizados por cada persona para lograr identidad, reconocimiento y presencia en el colectivo.

En la escuela se vivencian las relaciones de inclusión y de exclusión, en este último caso, se manifiesta la lucha contra los factores que generan marginamiento social. No obstante, aunque parte de la vida de los escolares se realiza al interior de las instituciones educativas, su vida cotidiana también se lleva a cabo en ambientes no escolarizados, es decir, son diferentes los escenarios donde se expresan sus intereses y motivaciones. Estas circunstancias inciden notablemente al momento de pensar y de ejercer la ciudadanía por parte de los jóvenes estudiantes.

\subsection{La condición social y cultural de la ciudadanía}

A pesar de que las sociedades contemporáneas evidencian serias exclusiones para diversos grupos de sus miembros, las cuales los llevan a la desesperanza y a la incertidumbre, al punto que muchos jóvenes que se piensan sin futuro, han confrontado los autoritarismos y la exclusión, y han construido manifestaciones de resistencia frente a los mismos. Por eso, se hace necesario identificar y comprender las diferentes formas como ellos configuran las ciudadanías, para generar acciones que contribuyan a hacer de nuestras sociedades mundos incluyentes donde haya presencia de los diferentes sectores de la población.

La escuela es uno de los escenarios donde se redescubren las diferentes expresiones de las ciudadanías, puesto que en ella los jóvenes vivencian su socialidad en las interacciones generadas en el ámbito educativo, crean nuevas formas de vivir la política, de asumir la institucionalidad, de tramitar los conflictos, de confrontar a otras generaciones, de vivir la sexualidad $y$, en general, de organizar la vida colectiva. En esta dirección, se puede afirmar que la escuela es un escenario importante donde se pueden identificar las maneras de resolver muchas dificultades que se derivan de las relaciones intersubjetivas, los procedimientos que permiten tramitar los conflictos interpersonales $y$ establecer pautas de convivencia que admitan la diversidad y fomenten la inclusión. 
En la escuela no sólo se construyen conocimientos relacionados con los significados y sentidos de la democracia, sino que se vivencian las diferentes expresiones de la ciudadanía. En ella, las nuevas generaciones descubren sus competencias y desempeños en relación con los asuntos de la vida colectiva y con el sentido de pertenencia, de identidad, y de construcción de los proyectos de sociedad en donde los significados y sentidos de vida de las personas se hallen presentes.

\subsection{La normatividad sobre ciudadanía es una oportunidad: el caso colombiano}

Con lo dicho, no es difícil imaginar que la institución escolar ejerza como un laboratorio de relacionamiento de los ciudadanos con las diferentes esferas de la realidad social, puesto que en la vida pública, las pautas de convivencia se acuerdan mediante procesos de negociación para establecer normas que reglamentan las interacciones de los sujetos entre sí y con la vida institucional.

En coherencia con lo anterior, la normatividad colombiana se preocupa por los asuntos de la educación para la democracia. En el artículo 41 de la Constitución Política de 1991, se ordena que: En todas las instituciones de educación, oficiales o privadas, serán obligatorios el estudio de la Constitución y la Instrucción Cívica. Así mismo se fomentarán prácticas democráticas para el aprendizaje de los principios y valores de la participación ciudadana y, de la misma manera, comprometen al Estado y a la sociedad en la perspectiva de garantizar la participación activa de los jóvenes en los organismos públicos y privados que tengan a cargo la protección, educación y progreso de la juventud (art. 45). De manera similar, y coherente con el espíritu constitucional, en el artículo 67, se dice que esta formación debe estar orientada hacia el respeto a los derechos humanos, a la paz y a la democracia.

En el mismo sentido, la Ley General de Educación (Ley 115 de 1994), en los objetivos de los diferentes niveles de formación, en la definición de las comunidades educativas, el gobierno escolar, en la reglamentación de los manuales de convivencia y en los Proyectos Educativos Institucionales, (PEI), se alude permanentemente a la necesidad de fomentar el espíritu ciudadano, desde perspectivas éticas y cívicas, y en 
el marco de la formación en el respeto a la vida y a los demás derechos humanos, a la paz, a los principios democráticos, de convivencia, pluralismo, justicia, solidaridad y equidad, así como en el ejercicio de la tolerancia y de la libertad (art. 5, num. 2).

\subsection{La perspectiva de los derechos ciudadanos en la escuela}

En las instituciones educativas, es usual que los jóvenes actúen en función de la democracia representativa, (mediante la cual los estudiantes delegan el poder y la capacidad de decisión), o de la democracia participativa, cuando ellos mismos asumen sus derechos, deberes y responsabilidades, haciendo uso de los espacios y mecanismos de participación que les otorga el gobierno escolar.

De esta manera, la elaboración de los PEI, puede generar una posibilidad de concertación puesto que al participar los estudiantes en la construcción colectiva del código de convivencia, ellos se hacen sujetos de las prácticas sociales educativas. Ello se complementa con el nombramiento democrático de sus representantes en el gobierno escolar, en las instancias directivas de las instituciones educativas y del personero, quien es el encargado de vigilar el cumplimiento de los derechos de los estudiantes. Éstas, entre otras prácticas institucionales, muestran cómo se favorece la perspectiva representativa de la democracia en la institución escolar. Aún no es claro, sin embargo, que la representatividad se encuentre acompañada de una perspectiva participativa, donde todos los estudiantes puedan poner su palabra en forma equitativa en las grandes decisiones de la institución.

\subsection{Posibilidades y oportunidades de ejercer la ciudadanía en la escuela}

En el ambiente educativo, los estudiantes desarrollan las habilidades y destrezas que requieren para actuar socialmente, la capacidad de reflexión, de crítica, el manejo de las obligaciones y responsabilidades para con el colectivo social. Bula (2002) señala cómo en la escuela se hacen presentes las titularidades, los derechos, las capacidades, las oportunidades y las libertades, condiciones que subyacen en el ejercicio de la ciudadanía.

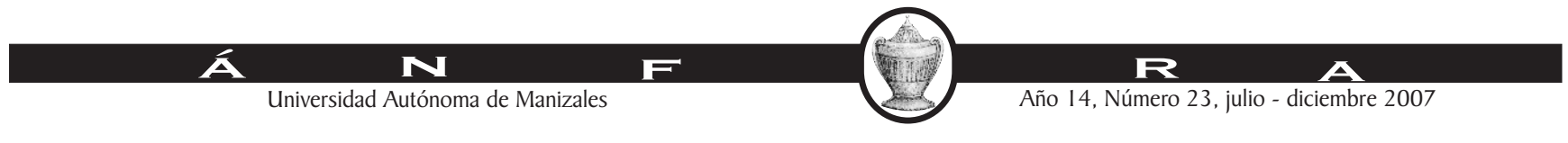


Las titularidades se entienden como el dominio que una persona tiene sobre un conjunto de bienes y servicios, en un espacio de reconocimiento y participación social. Algunas titularidades acompañan al individuo cuando nace y otras se adquieren a lo largo de la vida.

Las capacidades corresponden a las habilidades, condiciones y circunstancias que les permiten a las personas construir sus propios proyectos de vida y tener la libertad para decidirse por lo que más valoran. Las capacidades se asumen como los derechos reclamados, adquiridos y usados como fuente del poder de los sujetos, dado que con su aplicabilidad tienen la posibilidad de desarrollarse, tanto en lo personal como en lo social. El uso de las capacidades tiene relación directa con las oportunidades, y estas dependen de la vulnerabilidad.

La Vulnerabilidad tiene que ver con la cantidad y calidad de los recursos o activos, que controlan los individuos y las familias. En la medida en que las dotaciones iniciales sean disparejas, la capacidad de negociación es también dispareja y el acceso a las oportunidades es restringido.

La libertad corresponde a las posibilidades que tienen las personas para hacer uso de sus capacidades como humanos para alcanzar utilidades. Se trata de contribuir a la construcción de una sociedad que permita la expansión de las libertades y de brindarle la oportunidad a cada persona para que "moldee su propia vida". Una perspectiva de desarrollo humano en asuntos ciudadanos se orienta a buscar la expansión de las capacidades y las libertades de las personas para que puedan optar entre las distintas alternativas que le brinda la vida.

Además, la construcción de la ciudadanía toca con la calidad de vida y con el ejercicio de los derechos. Para ello, hay que contar con variables tales como el acceso al conocimiento, el desarrollo de capacidades y de potencialidades, los niveles de participación y de inserción social, el capital social, la generación e incremento de oportunidades.

En el mismo sentido, algunos componentes que limitan el desarrollo humano tienen que ver con la ausencia de medios adecuados de vida, de trabajo y de protección. Estos aspectos afectan dicho desarrollo, dado que obstaculizan el aprovechamiento de las capacidades, y como resultado afectan el ejercicio de la ciudadanía.

\section{A $\quad \mathbf{N}$}


En la escuela, son visibles las demandas y las respuestas que las instituciones educativas ofrecen a los diferentes actores que participan de las prácticas académicas, dado que se trata de que puedan conseguir las competencias que les permitan cualificar sus capacidades, lograr las titularidades, los derechos, las oportunidades y las libertades a que tienen derecho.

\subsection{Los retos del mundo escolar en la configuración de ciudadanos}

Las vivencias del mundo escolar están abocadas a enfrentar las distancias que se presentan entre la institucionalidad y los ciudadanos, para que se encuentren escenarios y mecanismos donde los sujetos puedan participar en el diseño y la ejecución de los asuntos que afectan sus vidas.

\section{Primer reto: lucha contra la exclusión en el conocimiento}

Los conocimientos, cada vez más se asumen como elementos determinantes para la configuración de las relaciones entre los sujetos y los mundos en los cuales ejercen su existencia. Sin duda, el fenómeno de la globalización, el acelerado proceso de individualización, los avances científico-tecnológicos, la revolución de la informática y de las comunicaciones, entre otros aspectos, han repercutido profundamente en las formas de ser ciudadano.

En ese sentido, uno de los principales desafíos del futuro, asociado a la transición de la sociedad de bienestar a la sociedad de la información, se relaciona con la producción, distribución, acceso y control de los conocimientos por parte de la ciudadanía. En palabras de Tedesco, "la pugna por apropiarse de los lugares donde se produce y se distribuye el conocimiento socialmente significativo, constituirá el centro de los conflictos sociales del futuro" (Tedesco, 1999).

Estratégicamente, las prácticas pedagógicas están llamadas a desarrollar los procesos de producción, intercambio y distribución de conocimiento y la preparación de ciudadanos, para que puedan hacer uso de éstos conocimientos y hacer posible su desarrollo personal en la perspectiva del bien común. 
De lo anterior, se puede colegir que el primer reto para la práctica educativa es la eliminación de la discriminación y la segregación social en el acceso al conocimiento, para que los jóvenes puedan acceder a las oportunidades sociales y participar activamente en las decisiones públicas y en el desarrollo de la sociedad.

\section{Segundo reto: mejoramiento de la calidad de la educación}

La masificación de la educación en términos de la ampliación cuantitativa, ha llevado a la discriminación social puesto que ha estimulado la creación de las escuelas pobres para pobres, que conducen a subvalorar las capacidades y los esfuerzos de quienes se forman en ellas. Al contrario, un reto del sistema educativo es buscar el mejoramiento de la calidad de la educación.

Se hace preocupante que en las acciones educativas se desvirtúe el acceso a una preparación que habilite a los jóvenes para desempeñarse con competencia en el mundo adulto. El desprecio por el esfuerzo, el rigor y la disciplina en las escuelas oficiales tiene consecuencias en el detrimento de la educación pública y permite la discriminación y el marginamiento de los estudiantes que se forman en ella.

Fanfani (2000), nos propone que una buena escuela para jóvenes debe tener, entre otras, las siguientes características:

a) Una institución abierta que valoriza y tiene en cuenta los intereses, expectativas de los jóvenes, se apoya en los conocimientos que poseen y que requieren.

b) Una escuela que favorece y estimula el protagonismo de los jóvenes y donde sus derechos y deberes se expresan en prácticas tangibles, y no sólo se enuncian en los programas y contenidos de documentos escolares.

c) Una institución que no se limita a enseñar sino que motiva, interesa, moviliza y desarrolla conocimientos significativos en la vida de las personas.

d) Una institución que se interesa por los jóvenes como personas totales e integrales que se desempeñan en diversos campos 
sociales (la familia, el barrio, el deporte, etc.) y no sólo los concibe como "alumnos" y/o "adolescentes".

e) Una institución flexible en tiempos, secuencias, metodologías, modelos de evaluación, sistemas de convivencia, etc. y que toma en cuenta la diversidad de la condición juvenil (de género, cultura, social, étnica, religiosa, territorial, etc.).

f) Una institución que forma personas y ciudadanos y no "expertos" es decir, que desarrolla competencias y conocimientos transdisciplinarios útiles para la vida, y no sólo disciplinas y conocimientos que sólo tienen valor en la escuela. ${ }^{5}$

g) Una institución que atiende a las diferentes dimensiones del desarrollo humano, y dónde los jóvenes aprenden a aprender en felicidad y que integra el desarrollo de la sensibilidad, la ética, la identidad y el conocimiento técnico-racional.

h) Una institución que acompaña y facilita la construcción de proyectos de vida de y para los jóvenes. Para ello deberá desplegarse una "pedagogía de la presencia" caracterizada por el compromiso, la apertura y la reciprocidad del mundo adulto para con los adolescentes y los jóvenes (GOMEZ DA COSTA A. C. 2000 y 1997)

i) Una institución que desarrolla el sentido de pertenencia y con la que los jóvenes "se identifican" y desarrollan el sentido de lo colectivo, es decir, del "nosotros".

\section{Tercer reto: formación para la ciudadanía.}

Por último, en relación directa con las pretensiones del presente trabajo, se destacan los aportes de Roggi (2004) sobre los nuevos ambientes en los cuales se realizan los procesos de formación en ciudadanía a partir de las aulas escolares, orientándola como un asunto de humanización en la perspectiva liberadora del hombre. En este sentido, la educación está abocada a desarrollar en los jóvenes estudiantes, entre otras, las siguientes capacidades:

Capacidad comunicativa: Preparar personas con competencias comunicativas tales como manejo adecuado del idioma nativo e incluso el aprendizaje de otros que les permitan acercarse a la comunidad
5 En otro lugar hemos criticado la tendencia a la organización disciplinaria del curriculum de la educación básica y el predominio del teoricismo, el esquematismo y la abstracción sin sentido en la enseñanza escolar (Cfr.: FANFANI E 2000).

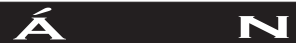

Universidad Autónoma de Manizales
$F$

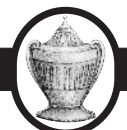

\section{$\mathbf{R}$}

Año I4, Número 23, julio - diciembre 2007 
internacional, lectura comprensiva, acceso a fuentes de información, contacto de trabajo con grupos de especialistas con los cuales puedan intercambiar informaciones y juicios. Se trata de que estas personas sean capaces de construir caminos colectivos que les permitan transitar la vida, y hacer realidad los futuros que sueñan y que sean posibles.

Preparación de ciudadanos: Contribuir a la formación de personas que se interesen por lo desconocido y lo ignorado, que sean curiosos, que se motiven por alcanzar conocimientos y que consideren que pueden lograrlos apoyados en sus capacidades, en sus esfuerzos y haciendo uso de métodos adecuados para ello. Ello requiere promover la conformación de grupos de estudio que permitan comprender y practicar la idea de que la construcción de conocimientos se hace con los demás. Para ello es importante que los ciudadanos se formen y se apoyen en marcos interpretativos que les permitan identificar, comprender, expresar y desempeñarse con los demas a partir de las condiciones reales que se viven en nuestros países.

Apertura a lo plural y a lo diferente: Desarrollar entre los estudiantes los valores de solidaridad, reconocimiento de las minorías, la denuncia de situaciones sociales y culturales de exclusión, para que puedan ampliar sus visiones del mundo en relación con el conocimiento $y$, con base en ello, lograr miradas de la realidad más amplias e incluyentes.

Análisis de las prácticas: Se trata de que los participantes de la vida escolar desarrollen competencias que les permitan revisar las acciones sociales de los diferentes grupos de población, para que estas puedan ser evaluadas y retroalimentarlas, buscando con ello, eficiencia y transparencia en el desarrollo de las mismas. Al respecto, desde la institución escolar, se puede y se debe hacer posible que los estudiantes logren aprendizajes de este tipo, que les acompañen durante su vida y sean la base de sus acciones sociales futuras.

La práctica de la acción colectiva: La institución escolar está en la necesidad de contribuir a la formación de sus jóvenes estudiantes para que puedan generar, organizar, concretar y evaluar acciones colectivas que les permitan resolver problemas comunes. Se trata de responder a las demandas que plantea la vida cotidiana en relación con las múltiples expresiones de asociación que le son exigidas a las personas para que

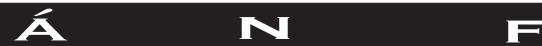

Universidad Autónoma de Manizales 
asuman compromisos, responsabilidades y realicen acciones colectivas que les lleve a desenvolverse adecuadamente al servicio del conjunto de la sociedad.

\section{BIBLIOGRAFÍA}

BULA J. I. (2002). Estado, Sociedad Civil y Democracia. Conferencia en: Seminario de Doctorado en "Ciencias Sociales". Manizales: febrero de 2002. S.P.

CASTILLO GARCÍA, José Rubén (2003). La formación de ciudadanos: la escuela, un escenario posible. En: Revista Latinoamericana de Ciencias Sociales, Niñez y Juventud. Centro de Estudios Avanzados en Niñez y Juventud, Vol. 1, No. 2, julio - diciembre de 2003. ISSN: $1692-715 X$. Manizales, Colombia.

\section{CONSTITUCIÓN POLÍTICA DE COLOMBIA DE 1991}

DURSTON J. (2000). Artículo publicado originalmente en la Revista Iberoamericana de Juventud $\mathrm{N}^{\circ} 1$, Madrid, 1996; editada por la Organización Internacional del Trabajo OIT. Todas las páginas web de este sitio fueron creadas por EAR y aprobadas por PDW. Copyright (C) 2000.

GIMENO SACRISTÁN, José. (2001). La cultura y la formación para la ciudadanía democrática. En: Educar y convivir en la cultura global. Capitulo IV. Editorial Morata, Madrid.

LEY 115 de 1994 (Ley General de Educación de la República de Colombia).

REGUILLO CRUZ, Rossana (2000). Emergencia de culturas juveniles. Estrategias del desencanto. Enciclopedia Latinoamericana de Sociocultura y Comunicación. Grupo editorial NORMA, Buenos Aires.

ROGGI, Luis Oswaldo (2004). Educación y construcción de ciudadanía o formación de nuevas ciudadanías. Conferencia en: Seminario de Doctorado en Ciencias Sociales. Niñez y juventud, Manizales: mayo de 2004. S.N.T. 
SANIN V.J. L.-Compilador- (1998) "Convivencia Escolar. Enfoques y Experiencias". Centro de Estudios Superiores Sociales y Políticos. CESEP. Medellín: Corporación Paisa Joven.

SANTA CRUZ G, Luis Eduardo (2005). Reflexiones críticas en torno a la formación ciudadana en la institución escolar. Documento PIIE. Programa interdisciplinario de investigaciones en educación. Chile,.

TENTI FANFANI, Emilio (2000). Culturas juveniles y cultura escolar. Buenos Aires, mayo de 2000. Documento presentado al seminario "Escola jovem: un nuevo olhar sobre o ensino médio. Organizado por el Ministerio de Educacao. Secretaria de Educacao Media e Tecnológica. Coordenacao-Geral de Ensino, Brasilia, del 7 al 9 de junio de 2000. 